\title{
Photoelectron imaging of guinea-pig, hamster and human spermatozoa
}

\author{
R. Mrsny and O. H. Griffith \\ Institute of Molecular Biology and Department of Chemistry, University of Oregon, Eugene, \\ Oregon 97403, U.S.A.
}

\begin{abstract}
Summary. Photoelectron images of mammalian spermatozoa were obtained by subjecting the specimens to u.v.-irradiation and focussing the emitted electrons by electron optics (photoelectron microscopy). Guinea-pig, hamster and human spermatozoa were fixed in glutaraldehyde, deposited on conductive glass discs, and dehydrated. Sufficient quantities of photoelectrons were released from the surface of spermatozoa to produce images without staining, coating or metal shadowing. The large planar heads of guinea-pig spermatozoa were easily resolved with good delineation of acrosomal and postacrosomal regions. Residual vesicles could be visualized on the surface of the inner acrosomal membrane of spermatozoa that had undergone the acrosome reaction. Also detectable in these photoelectron images were finer membrane surface details, periodicities in the midpiece region of the tail which coincided with the distribution of mitochondria, and periodicities in the principal piece which appeared to be related to fibrous sheath components. Hamster spermatozoa were similarly well resolved but human spermatozoa were more difficult to image because of their increased surface curvature. The mechanism responsible for detection of these surface details is primarily topographical contrast rather than material contrast, since spermatozoa coated with a thin layer of gold or platinum exhibited similar features, although at reduced resolution, as the uncoated specimens.
\end{abstract}

\section{Introduction}

Photoelectron imaging can be thought of as the electron optical analogue of fluorescence microscopy (Griffith et al., 1972). Both techniques use an ultraviolet (u.v.) light source for excitation. In fluorescence microscopy, photons given up by molecules capable of fluorescence are imaged with light optics to provide a pattern of fluorescent molecules. Most biological molecules are capable of photoemitting electrons after u.v. excitation. Photoelectron imaging uses electron optics to accelerate, focus and image these emitted electrons to produce a representation of the specimen. Photoelectron imaging is also called photoelectron microscopy (PEM) in the United States and photoemission electron microscopy in Europe (PEM or PEEM). Its origins can be traced, along with those of both transmission and scanning electron microscopy, to the early 1930s (Mollenstedt \& Lenz, 1963; Schwarzer, 1981), but photoelectron microscopy has only recently been adapted for the imaging of biological specimens (Griffith et al., 1972) and it is best described as an emerging technique, rather than an established method. Of particular interest is the imaging of cell surfaces by this technique. Photoelectron images of whole cells obtained to date include those of several lines of cultured fibroblasts, epithelial cells, and red cell ghosts (for reviews see Griffith, Rempfer \& Nadakavukaren, 1982; Griffith, Nadakavukaren \& Jost, 1984). Spermatozoa have not been examined in detail. One early report contains a photoelectron micrograph of rabbit spermatozoa which showed that the general shape of this cell type could be visualized by photoelectron microscopy (Grund, Eichberg \& Engel, 1979). In principle, the photoelectron image 
of spermatozoa contains useful information regarding fine topographical detail and organization of the cell surface.

The purpose of the present report is to obtain higher resolution photoelectron images of the spermatozoa of several species of mammal.

\section{Materials and Methods}

Media. The minimal capacitation medium with lactate and pyruvate (MCM-PL) prepared was a slight modification of a $\mathrm{Ca}^{2+}$-free medium used by Hyne \& Garbers (1982). MCM-PL consisted of $126 \mathrm{~mm}-\mathrm{NaCl}, 1 \mathrm{~mm}-\mathrm{MgCl}_{2}, 25 \mathrm{mM}-\mathrm{NaHCO}_{3}, 0.25 \mathrm{~mm}$-sodium pyruvate and $20 \mathrm{~mm}-\mathrm{L}(+)$ lactic acid (neutralized with $\mathrm{NaOH}$ ) at $295 \mathrm{mosmol} / \mathrm{kg}$ and $\mathrm{pH} 7 \cdot 4$. For capacitation, $\mathrm{CaCl}_{2}$ was added to Medium MCM-PL to a final concentration of $1.7 \mathrm{mM}$. Phosphate-buffered saline with sucrose (PBS-S) and modified Tyrode's bicarbonate buffer (TALP) used for capacitation were prepared essentially as described by Mrsny \& Meizel (1981). Medium PBS-S consisted of $72 \mathrm{mM}-\mathrm{NaCl}, 4 \cdot 25$ mM-K Cl, $3.2 \mathrm{~mm}-\mathrm{Na}_{2} \mathrm{HPO}_{4}, 0.8 \mathrm{mM}-\mathrm{KH}_{2} \mathrm{PO}_{4}, 0.46 \mathrm{~mm}-\mathrm{CaCl}_{2}, 0.2 \mathrm{mM}-\mathrm{MgCl}_{2}$ and $132 \mathrm{~mm}$-sucrose at $290 \mathrm{mosmol} / \mathrm{kg}$ and $\mathrm{pH} \mathrm{7.3.} \mathrm{Medium} \mathrm{TALP} \mathrm{contained} 121 \mathrm{~mm}-\mathrm{NaCl}, 0.4 \mathrm{~mm}-\mathrm{NaH}_{2} \mathrm{PO}_{4}, 5 \mathrm{~mm}-$ $\mathrm{KCl}, 25 \mathrm{~mm}-\mathrm{NaHCO}_{3}, 2.4 \mathrm{~mm}-\mathrm{CaCl}_{2}, 0.4 \mathrm{~mm}-\mathrm{MgCl}_{2}, 5 \mathrm{~mm}$-glucose, $12.5 \mathrm{~mm}-\mathrm{L}(+)$ lactic acid (neutralized with $\mathrm{NaOH}$ ), $0.25 \mathrm{~mm}$-sodium pyruvate, $12 \mathrm{mg}$ bovine serum albumin $/ \mathrm{ml}$ (purified by trichloroacetic acid precipitation, ethanol resolubilization and extensive dialysis against $\mathrm{H}_{2} \mathrm{O}$ and PBS) and $0.5 \mathrm{~mm}$-taurine. Hepes-buffered saline (HBS) consisted of $50 \mathrm{~mm}-\mathrm{Hepes}, 3.4 \mathrm{mM}-\mathrm{CaCl}_{2}$, 6.7 mM-KCl, 0.6 mM- $\mathrm{MgSO}_{4}$ and $137 \mathrm{~mm}-\mathrm{NaCl}$ at $310 \mathrm{mosmol} / \mathrm{kg}$ and at $\mathrm{pH} 7 \cdot 2$. All solutions were passed through $0.22 \mu \mathrm{m}$ Millex-GS filter units (obtained from Millipore) before use.

Preparation of spermatozoa. Sexually mature guinea-pigs (Topeka strain) were anaesthetized with ether and killed by cervical dislocation. Cauda epididymal spermatozoa, obtained from punctured tubules, were layered on $10 \mathrm{ml}$ of Medium MCM-PL or HBS. Spermatozoa were washed twice by centrifugation $(500 \mathrm{~g}$ for $15 \mathrm{~min}$ ) and resuspended in the buffer used initially, MCM-PL or HBS. In some instances, after the second centrifugation in Medium MCM-PL, spermatozoa were resuspended in Medium MCM-PL containing $\mathrm{Ca}^{2+}$ at $5 \times 10^{6} / \mathrm{ml}$. To initiate capacitation, $0 \cdot 1 \mathrm{ml}$ aliquants of this sperm suspension were incubated at $37^{\circ} \mathrm{C}$.

Sexually mature golden hamsters were anaesthetized with ether and killed by cervical dislocation. Spermatozoa obtained from punctured tubules of the cauda epididymidis were layered on $10 \mathrm{ml}$ of Medium PBS-S or HBS. Spermatozoa were washed twice by centrifugation ( $800 \mathrm{~g}$ for 10 min) and resuspended in the buffer used initially, PBS-S or HBS. In some instances, after the second centrifugation, spermatozoa, washed in Medium PBS-S were resuspended in Medium TALP at $5 \times 10^{6} / \mathrm{ml}$. To initiate capacitation, spermatozoa were incubated in $0.1 \mathrm{ml}$ volumes at $37^{\circ} \mathrm{C}$.

Semen from a healthy man of known fertility was obtained after $48 \mathrm{~h}$ of abstention. The semen was allowed to liquefy at room temperature for $1 \mathrm{~h}$, and aliquants $(0.5 \mathrm{ml})$ were then placed under 5 $\mathrm{ml}$ warm Medium HBS and incubated for $2 \mathrm{~h}$ at $37^{\circ} \mathrm{C}$. The spermatozoa that had swum into the Medium HBS were washed twice in Medium HBS ( $500 \mathrm{~g}$ for $15 \mathrm{~min}$ ) and resuspended in Medium HBS.

Assay of sperm motility and acrosome reaction. Samples $(\sim 50 \mu \mathrm{l})$ were removed from suspensions of washed guinea-pig and hamster spermatozoa or from suspensions of incubated spermatozoa and examined by phase-contrast and dark-field microscopy. Estimates of motility and hyperactivated motility (whiplash-like flagellar movement characteristic of capacitation in some spermatozoa) were assessed according to previously described criteria (Yanagimachi, 1969, 1970, 1972). An objective count was performed of at least 100 motile cells to determine the percentage of cells that had undergone the acrosome reaction. No attempt was made to capacitate the human spermatozoa. 
Sperm preparation for photoelectron microscopy. Round glass coverslips $(5 \mathrm{~mm})$ were used as sample mounts after being first made conductive with a layer of $\mathrm{SnO}_{2}$ (Griffith et al., 1982). Adhesion of cells to these discs was facilitated by dipping the discs in $1 \mathrm{mg} / \mathrm{ml}$ aqueous solutions of alcian blue, cytochrome c, or poly-L-lysine (6000 average mol. wt, brought to $\mathrm{pH} 13$ with $\mathrm{NaOH}$ ) and air-drying. Washed spermatozoa were affixed to coated glass discs by exposure for $15 \mathrm{~min}$, with excess spermatozoa being drawn off. Adhering spermatozoa were fixed for $1 \mathrm{~h}$ in $2.5 \%$ glutaraldehyde at room temperature and the discs were washed with $\mathrm{H}_{2} \mathrm{O}$. Spermatozoa incubated for capacitation were collected, washed once in Medium MCM (guinea-pig) or Medium PBS (hamster) and fixed as above. No differences were observed in preparations when the sperm cells were fixed before placement on the glass discs. Post-fixation with $1 \% \mathrm{OsO}_{4}$ for $1 \mathrm{~h}$ at room temperature provided no improvement of the photoelectron images.

For photoelectron microscopy, the fixed cells were dehydrated through a graded series of ethanol (acetone produced equivalent results) followed by air-drying. Replacement of ethanol with freshly distilled Freon 113 followed by air-drying frequently caused loss of tail and some head membranes of spermatozoa. After dehydration through ethanol, some specimens were criticalpoint dried from $\mathrm{CO}_{2}$ but judged to be inferior due to shrinkage artefacts.

After ethanol dehydration and air-drying, some specimens were then over-coated with a $15 \mathrm{~nm}$ layer of gold or platinum in a Varian FC-12 ultrahigh vacuum chamber (metal-sealed, oil-free). Current was passed through a loosely coiled tungsten filament wrapped with a 26-gauge wire composed of gold or platinum (wires were rinsed and stored in distilled acetone) which was positioned directly above the specimens at a distance of $10 \mathrm{~cm}$ to minimize shadowing. The rotoevaporated metal film thickness was monitored using an Inficon XTM quartz crystal oscillator which had been previously calibrated with an interference microscope (Varian A-scope).

The photoelectron microscope used in this study is an ultrahigh vacuum instrument built at the University of Oregon (Griffith, Rempfer \& Lesch, 1981). The u.v.-illumination sources were two Osram HBO 100 short-arc mercury lamps. Accelerating voltage was $30 \mathrm{kV}$. Photoelectron micrographs were taken on Kodak 4489 electron microscopy film with exposure times of $10-30 \mathrm{sec}$ for uncoated specimens and 1-5 sec for gold- or platinum-coated samples. All micrographs were taken using a $50 \mu \mathrm{m}$ objective aperture.

\section{Results}

\section{Guinea-pig}

Photoelectron images of guinea-pig spermatozoa are shown in Plates 1 and 2. The acrosomal cap of the sperm head in Pl. 1, Fig. 2 was representative of many relatively flat spermatozoa

\section{PLATES $1-4$}

Abbreviations: AC, acrosomal cap; $C$, capitulum; CD, cytoplasmic droplet; EP, end piece of tail; ES, equatorial segment; $H$, head; IF, implantation fossa ; IM, inner acrosomal membrane; MP, midpiece of tail; $N$, neck; $P$, perforatorium; PA, postacrosomal region; $P P$, principal piece of tail; SC, segmented column.

\section{PLATE 1}

Fig. 1. Photoelectron micrograph of an entire guinea-pig spermatozoon. The morphological regions of this cell are identified. Bar, $5 \mu \mathrm{m} . \times 1200$.

Fig. 2. Higher magnification of a guinea-pig spermatozoon showing greater detail of head and neck regions. Note the topographical relief of the anterior acrosomal region (acrosomal cap) caused by the non-symmetric nature of its contents. Also note the ridge caused by a perinuclear thickening at the anterior margin (arrow heads) of the equatorial segment. The topographical contrast at the posterior margin (arrows) of the equatorial segment is caused by the thickening of the cell where the acrosomal membrane folds back onto itself. Bar, $2 \mu \mathrm{m} . \times 8500$. 
Fig. 3. Early stage of the guinea-pig sperm acrosome reaction induced by incubation in Medium MCM-PL with $\mathrm{Ca}^{2+}$ for $2.5 \mathrm{~h}$. Fenestrations of the plasma membrane at the anterior edge of the acrosomal cap and the area immediately anterior to the crescent-shaped margin of the nucleus are identified by arrows. Bar, $2 \mu \mathrm{m}$. $\times 5000$.

Fig. 4. Late stage of the guinea-pig sperm acrosome reaction induced by incubation for $2.5 \mathrm{~h}$ in Medium MCM-PL with $\mathrm{Ca}^{2+}$. Note the vesicles (arrows) present on the surface of the inner acrosomal membrane. A lip of topographical contrast at the rostral edge of the equatorial segment (arrow heads) marks the line of fusion between the remaining outer acrosomal membrane and plasma membrane. The perforatorium is observable at this stage of the acrosome reaction. Bar, $2 \mu \mathrm{m} . \times 5000$.

Fig. 5. Photoelectron micrograph of an acrosome-reacted guinea-pig spermatozoon following loss of acrosome reaction vesicles. This spermatozoon was prepared as in Pl. 1, Figs 1-4 except that critical-point drying was performed rather than air drying. Note the few remaining vesicles (arrows) and complete exposure of the inner acrosomal membrane anterior to the equatorial segment. The ridge of fusion between the remaining outer acrosomal membrane and plasma membrane (arrow heads) is still evident. Bar, $2 \mu \mathrm{m} . \times 5000$.

\section{PLATE 2}

Fig. 6. Photoelectron micrograph of the posterior head region, neck and proximal midpiece region of a guinea-pig spermatozoon. Note the prominent ridge produced by the implantation fossa (IF) at the head-neck junction. The segmented columns (SC) of the connecting piece are also observable. The midpiece shows an irregular pattern of periodicity representing mitochondria. Bar, $1 \mu \mathrm{m} . \times 7000$.

Fig. 7. Head, neck and midpiece regions of guinea-pig spermatozoon showing neck surface topography due to capitulum and prominent cytoplasmic droplet (CD). The cytoplasmic droplet was observed at various positions along the midpiece and was absent in most spermatozoa after capacitation. Bar, $1 \mu \mathrm{m} . \times 7500$.

Fig. 8. Principal piece region of a guinea-pig spermatozoon. The principal piece showed ridges and a partly resolved transverse periodicity representing the longitudinal columns (arrow) and ribs (arrow heads) of the fibrous sheath, respectively, which lie beneath the plasma membrane. Bar, $0.5 \mu \mathrm{m} . \times 17000$.

Fig. 9. Principal piece of guinea-pig spermatozoon prepared as in Fig. 8 except for replacement of ethanol with Freon 113 before air drying. Note the increased topographical resolution (compared with Fig. 8) of the longitudinal column (arrow) and ribs (arrow heads) of the fibrous sheath due to removal of the plasma membrane. In the lower portion of the micrograph the tail has twisted through $180^{\circ}$. Bar, $1 \mu \mathrm{m} . \times 12000$.

Fig. 10. Distal portion of guinea-pig sperm tail showing transition from principal piece to end piece. Bar, $0.5 \mu \mathrm{m} . \times 11000$.

Fig. 11. Distal portion of guinea-pig sperm tail prepared as in Fig. 10 except for replacement of ethanol with Freon 113 before air drying. Note the detailed resolution of the microtubules (arrows) in a frayed pattern following disruption of the axoneme due to loss of the plasma membrane. Bar, $1 \mu \mathrm{m}$. $\times 12500$.

Fig. 12. Photoelectron micrograph of guinea-pig spermatozoon with an intact acrosome after coating with a thin layer of platinum. Note the decreased surface detail in comparison with $\mathrm{Pl}$. 1, Fig. 2. Bar, $2 \mu \mathrm{m} . \times 5000$.

Fig. 13. Platinum-coated guinea-pig spermatozoon which has undergone the acrosome reaction. The line of fusion between remaining outer acrosomal and plasma membranes is identified by arrow heads. Bar, $2 \mu \mathrm{m}$. $\times 5000$.

Fig. 14. Photoelectron micrograph of a guinea-pig spermatozoon which has undergone the acrosome reaction. The sample has been coated with a thin layer of gold. The line of fusion between remaining outer acrosomal membrane and plasma membrane is identified by arrow heads. Bar, $2 \mu \mathrm{m}$. $\times 5000$. 

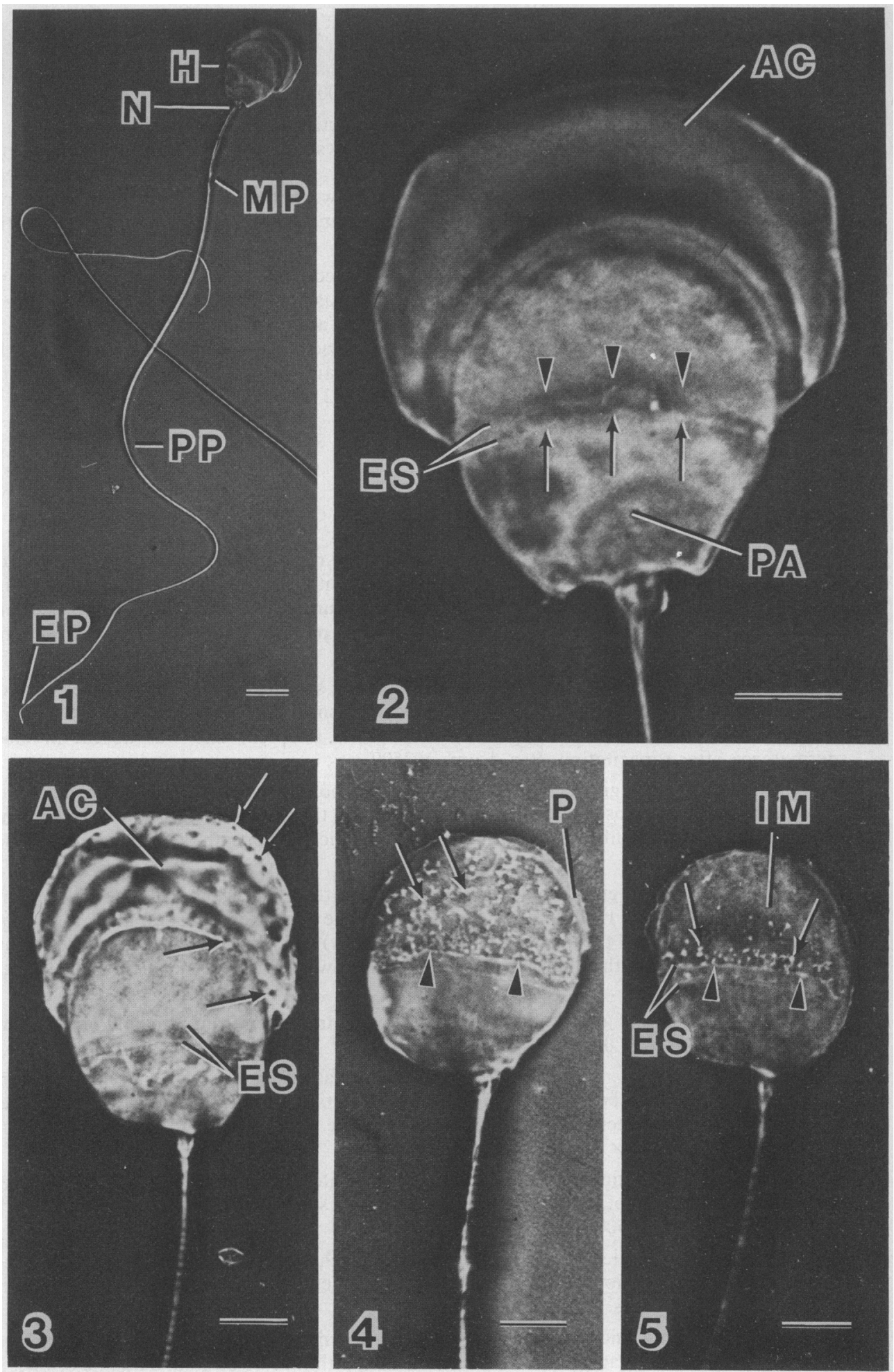

(Facing p. 130) 
PLATE 2

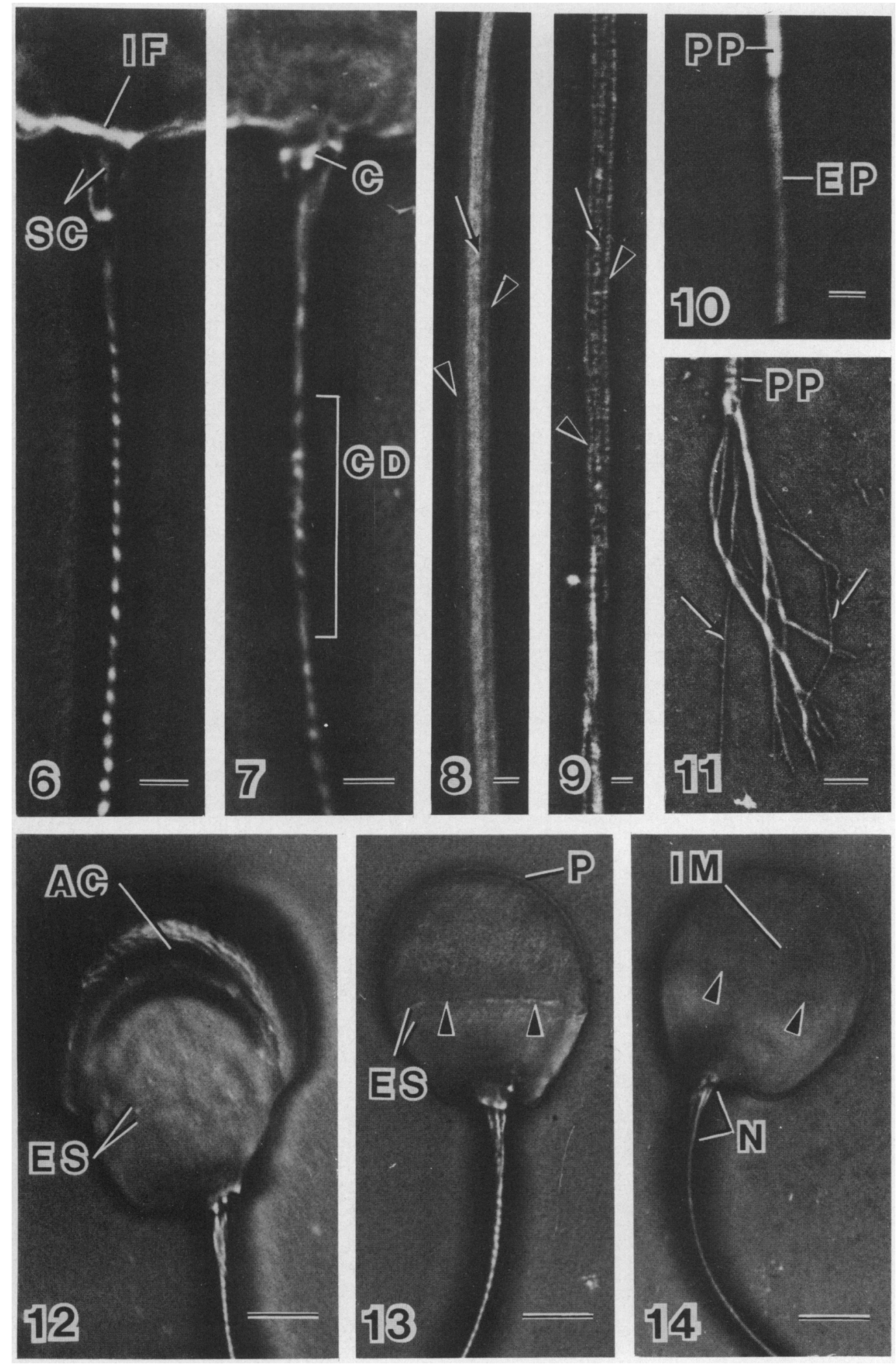


PLATE 3
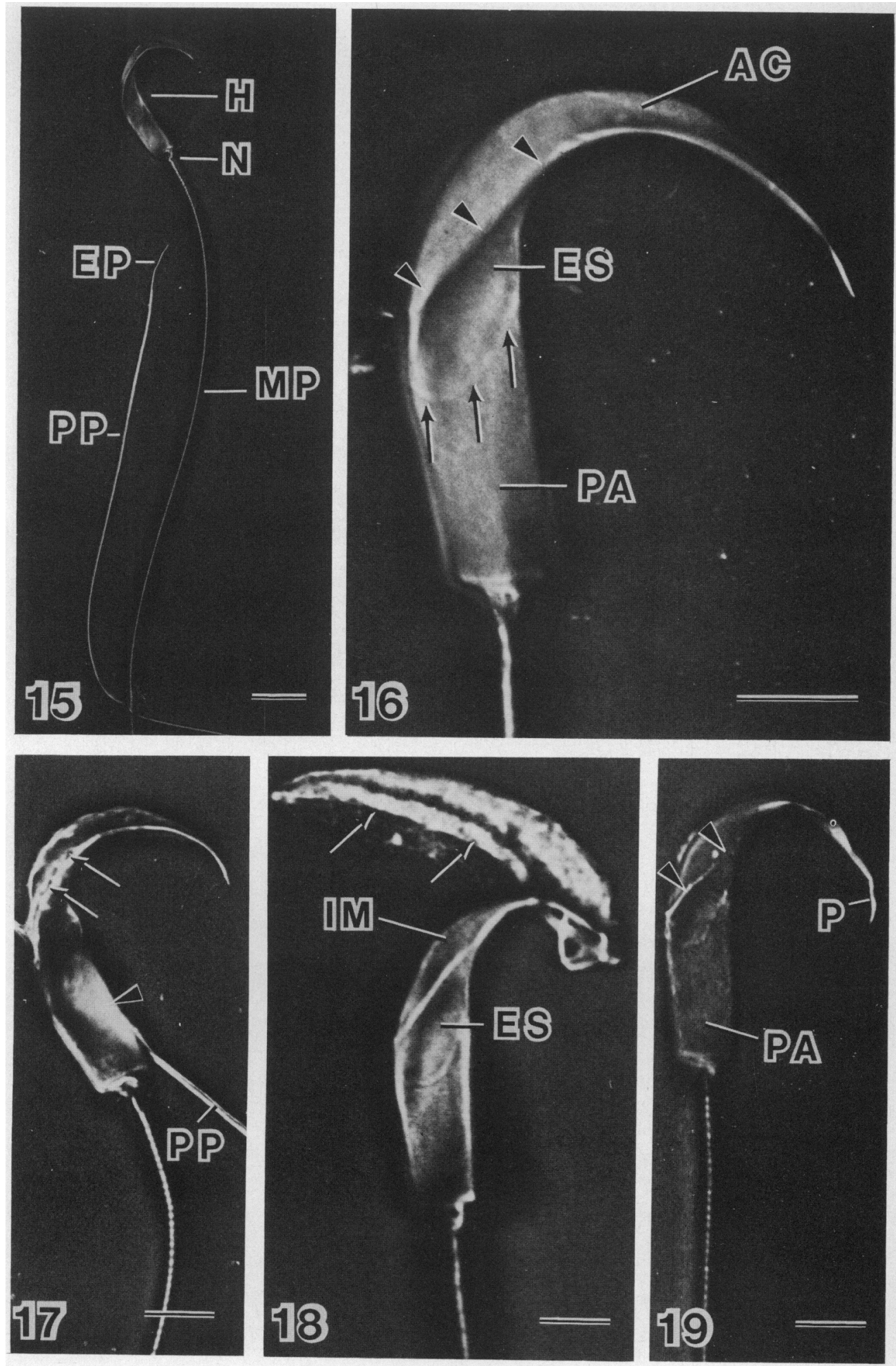
PLATE 4
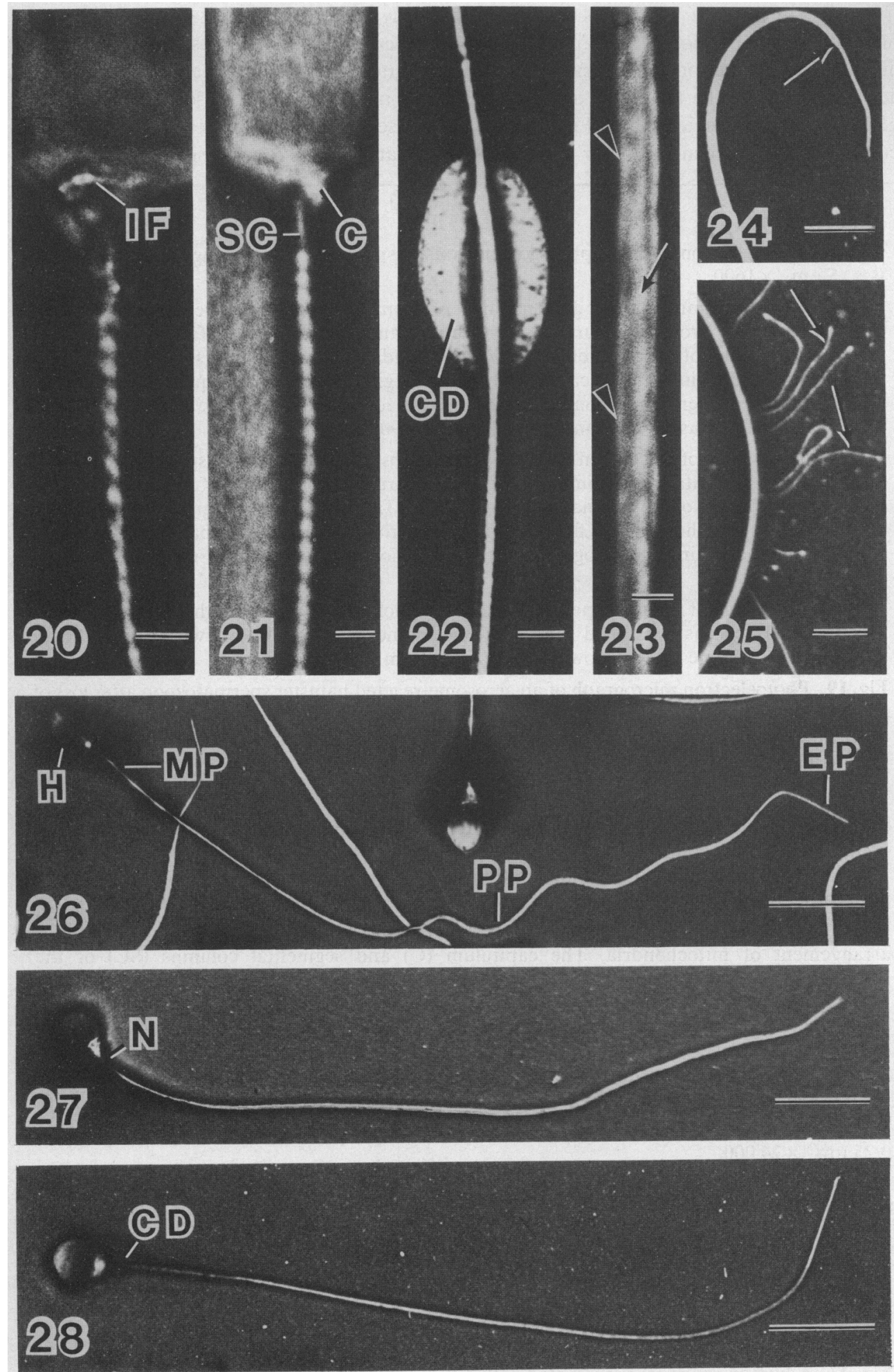
observed. There was a gradation of surface topography with some spermatozoa exhibiting an undulated acrosomal region. Pl. 1, Figs 3-5 illustrate sperm heads in various stages of acrosomal loss induced by incubation for several hours in a capacitating medium. The fenestrations of the surface membrane at the tip of the acrosomal cap and adjacent to the crescent-shaped anterior margin of the nuclear region (P1. 1, Fig. 3) have been observed previously as the initial fusion and vesiculation sites between the outer acrosomal membrane and its overlying plasma membrane

\section{PLATE 3}

Fig. 15. Photoelectron micrograph of golden hamster spermatozoa, showing the head and tail. Bar, $5 \mu \mathrm{m} . \times 1600$.

Fig. 16. Higher magnification of golden hamster spermatozoon showing greater detail of head regions. Note the prominent curved shape (broad anterior margin) of the acrosome. The topographical relief of the anterior margin (arrow heads) of the equatorial segment is due to a thickening of the perinuclear theca. The posterior margin (arrows) of the equatorial segment is observed as the topographical contrast caused by the acrosomal membrane folding back onto itself beneath the plasma membrane. Bar, $2 \mu \mathrm{m} . \times 9000$.

Fig. 17. Early stage of the golden hamster sperm acrosome reaction. The specimen had been incubated in a capacitating medium for $4 \mathrm{~h}$. Note the ruffled appearance of the acrosomal cap and the fenestrations of the plasma membrane (arrows) at the anterior margin of the equatorial segment. The sperm head in this field is lying atop (arrow head) the principal piece of another spermatozoon, affecting the topography (bright area) of its postacrosomal region. Bar, $2 \mu \mathrm{m}$. $\times 5000$

Fig. 18. Late stage of hamster sperm acrosome reaction. The acrosomal ghost (arrows) has a foamy appearance as it is shed during the acrosome reaction. The newly exposed inner acrosomal membrane (IM) is now evident. Bar, $2 \mu \mathrm{m} . \times 5000$.

Fig. 19. Photoelectron micrograph of an acrosome-reacted hamster spermatozoon after loss of the acrosomal ghost. Note the long perforatorium (formed by an extension of the perinuclear theca) and the fusion point of the remaining outer acrosomal membrane and plasma membrane (arrow heads). Bar, $2 \mu \mathrm{m} . \times 5000$.

\section{PLATE 4}

Fig. 20. Photoelectron micrograph of the distal head, neck and proximal midpiece of a golden hamster spermatozoon. The neck region shows topographical relief suggesting the subplasmalemmal structure of the implantation fossa (IF). This midpiece shows an irregular pattern of periodicity representing mitochondrial deposition. Bar, $0.5 \mu \mathrm{m} . \times 16000$.

Fig. 21. A golden hamster spermatozoon showing the midpiece periodicity of a helical arrangement of mitochondria. The capitulum (C) and segmental columns (SC) of the connecting piece are also observable. Bar, $0.5 \mu \mathrm{m} . \times 12000$.

Fig. 22. A golden hamster spermatozoon showing a prominent cytoplasmic droplet approximately half-way down the midpiece region of the tail. Cytoplasmic droplets were observed at various positions along the midpiece but were less frequently observed after several hours in a capacitating medium. Bar, $1 \mu \mathrm{m} . \times 7000$.

Fig. 23. Principal piece region of a hamster spermatozoon. The topographical contrast represents the longitudinal column (arrow) and ribs (arrow heads) of the fibrous sheath. Bar, $0.25 \mu \mathrm{m} . \times 24000$.

Fig. 24. Distal portion of a hamster sperm tail showing the transition (arrow) from the principal piece of the distal end piece. Bar, $1 \mu \mathrm{m} . \times 10000$.

Fig. 25. Distal portion of the principal piece of a hamster sperm tail. In this instance, membrane damage had occurred as assessed by the extrusion of fine filaments (arrows) which are probably components of the tail motility apparatus. Bar, $1 \mu \mathrm{m} . \times 9500$.

Fig. 26. Photoelectron micrograph of human spermatozoa depicting regions of the head and tail. Bar, $5 \mu \mathrm{m}$. $\times 3000$.

Fig. 27. A human spermatozoon coated with a thin layer $(15 \mathrm{~nm})$ of gold. Bar, $5 \mu \mathrm{m} . \times 3000$.

Fig. 28. Platinum-coated (15 nm thickness) human spermatozoon. Bar, $5 \mu \mathrm{m} . \times 3000$. 
during an early stage of the acrosome reaction (Friend, Orci, Perrelet \& Yanagimachi, 1977). Acrosomal vesicles, hybrid vesicles composed of both outer acrosomal and plasma membranes, are formed by the multiple fusion and vesiculation events of the acrosome reaction (Meizel, 1984). These vesicles were present on the surface of the newly exposed inner acrosomal membrane at late stages of the acrosome reaction (Pl. 1, Fig. 4). At such a stage of the acrosome reaction the perforatorium was clearly visible and observation of the fusion between the remaining outer acrosomal membrane and plasma membrane at the anterior edge of the equatorial segment was possible. Following loss of these vesicles, a clear view of the inner acrosomal membrane was attainable (Pl. 1, Fig. 5).

The periodic bright and dark bands in the midpiece region, which began posterior to the connecting piece and stopped at the end of the midpiece (the annulus), were irregular in their disposition, but the distance between adjacent bright bands was about $0.3 \mu \mathrm{m}$. This periodicity coincides with the distribution of mitochondria in mammalian spermatozoa (Fawcett, 1975). Coating of spermatozoa with a thin layer of metal increased the brightness of the photoelectron images, but reduced the resolution of the fine surface detail (Pl. 2, Figs 12-14).

\section{Hamster}

Photoelectron micrographs of golden hamster spermatozoa are shown in Plates 3 and 4 . The very different head morphology of these cells provides a separate test of photoelectron imaging. The equatorial segment of the head was delineated by topographical relief at its margins. The anterior margin is the ridge of thickened perinuclear material (part of the theca) and the posterior margin being the site of reflection of the acrosomal membrane (Franklin, Barros \& Fussell, 1970). Golden hamster spermatozoa showed no variation in topography of the intact acrosomal cap region (Pl. 3, Figs 15, 16). During the acrosome reaction the acrosome was foamy in appearance and the acrosomal ghost (Yanagimachi \& Phillips, 1984) lifted away to expose the inner acrosomal membrane (Pl. 3, Figs 17-19).

The periodicity of the golden hamster sperm midpiece region showed an irregular (P1. 4, Fig. 20) or a helical (Pl. 4, Fig. 21) pattern of banding.

\section{Human}

Human spermatozoa were more difficult to image by photoelectron microscopy (Pl. 4, Fig. 26) than were guinea-pig or hamster spermatozoa. Even under the best conditions, some image distortion occurred over the human sperm head. Coating with a thin layer of gold or platinum did not dramatically reduce the image distortion which must therefore have been due to the topography of the spermatozoa and not specimen charging. Those spermatozoa that could be viewed, however, did show the morphological variation of the sperm head observed in this species (Bedford, Bent \& Calvin, 1973). As with guinea-pig and hamster spermatozoa, human sperm tail structures (midpiece, principal piece and end piece) were well imaged (Pl. 4, Figs 26-28).

\section{Discussion}

Photoelectron imaging is still under development as a technique for relating ultrastructure to cell function. The present results demonstrate the application of photoelectron imaging at its present state of development for the study of the surfaces of mammalian spermatozoa. 
The photoelectron image is formed by collecting all of the electrons released from the surface at the same time rather than point by point as in a scanning electron microscope. Two sources of contrast, material and topographical, can contribute to the photoelectron image of a cell surface (Rempfer, Nadakavukaren \& Griffith, 1980; Griffith et al., 1982). Material contrast is not a major contributor to these photoelectron images of spermatozoa, as judged by comparing the uncoated and metal coated specimens. The image is sensitive to very fine topographic detail because the low energy electrons emitted from the surface are readily influenced by the accelerating field across the cathode-anode gap. Electrons emitted from sloping surfaces of protrusions or depressions are deflected, making it possible to detect very small cell surface features in the image. It is this topographical contrast mechanism that gives the photoelectron micrographs their three dimensional appearance. No image processing is required. The topographical and other image information is recorded directly onto a photographic film as in transmission electron microscopy. In these photoelectron micrographs the major known structural features of the mammalian spermatozoon, such as the acrosomal cap, equatorial segment, postacrosomal region, neck, midpiece, principal piece, and end piece are well resolved and fine surface detail is visible. In theory, very small steps of surface topography can be detected by this approach.

The high sensitivity to topographic contrast places a limit on the range of topography that can be accommodated by this technique. The heads of human spermatozoa are at the limit of this range because they are more rounded than are the guinea-pig or hamster sperm heads. All of the major known topographic features of guinea-pig and hamster spermatozoa are readily identified in the photoelectron images which provide a faithful representation of the surface and correlate well with the morphology previously established by other microscopic techniques such as optical microscopy, thin section and replica transmission electron microscopy and scanning electron microscopy.

Each microscopic technique has a different physical basis for image formation and therefore provides a different information content. For example, the energy source for photoelectron production, the u.v. light, penetrates deeply into biological specimens. However, these low energy electrons are easily scattered by organic material and the electrons actually escaping and contributing to the photoelectron image originate primarily in the top surface layer (Houle, Engel, Willig, Rempfer \& Griffith, 1982). This is normally the plasma membrane unless it is disrupted or removed as, for example, a consequence of the acrosome reaction. As a result of the short escape depth of the photoelectrons the surface detail is sharply imaged. This has been described as a high depth of resolution or a short depth of information (Houle et al., 1982). Objects beneath the surface can also contribute to the image by inducing topography at the surface. For example, marking the rostral edge of the equatorial segment of spermatozoa is a ridge of the perinuclear theca which is beneath the plasma membrane, cytoplasm, outer acrosomal membrane, acrosomal matrix, and inner acrosomal membrane (Fawcett, 1975). This ridge is observed in the photoelectron micrographs (Pl. 1, Fig. 2; Pl. 3, Fig 15) not because the electrons forming the image originate from this perinuclear material but because the plasma membrane and underlying membranes are draped over it, producing an elevation at the surface. Another example is provided by the photoelectron images of the midpiece region. The observed periodicity is a result of the plasma membrane draped over the mitochondria which are known to be circumferentially orientated around the core complex in a helical or irregular pattern (Phillips, 1977).

As the techniques of photoelectron imaging and photoemissive marker technology are developed further they may contribute to our understanding of the molecular events of fertilization by allowing the locations of specific cell surface components of the spermatozoon to be mapped.

We thank Douglas Habliston for the photoelectron micrographs, Lori Evans for preparation of the figures; and Walter Skoczylas and Dr Gertrude F. Rempfer for continued improvement to the University of Oregon photoelectron microscope. This work was supported by PHS Grant CA 11695. R.J.M is the recipient of NIH postdoctoral fellowship GM 08712. 


\section{References}

Bedford, J.M., Bent, M.J. \& Calvin, H. (1973) Variations in the structural character and stability of the nuclear chromatin in morphologically normal human spermatozoa. J. Reprod. Fert. 33, 19-29.

Fawcett, D.W. (1975) The mammalian spermatozoon. Devl Biol. 44, 394-436.

Franklin, L.E., Barros, C. \& Fussell, E.N. (1970) The acrosomal region and the acrosome reaction in sperm of the golden hamster. Biol. Reprod. 3, 180-200.

Friend, D.S., Orci, L., Perrelet, A. \& Yanagimachi, R. (1977) Membrane particle changes attending the acrosome reaction in guinea pig spermatozoa. $J$. Cell Biol. 74, 561-577.

Griffith, O.H., Lesch, G.H., Rempfer, G.F., Birrell, G.B., Burke, C.A., Schlosser, D.W., Mallon, M.H., Lee, G.B., Stafford, R.G., Jost, P.C. \& Marriott, T.B. (1972) Photoelectron microscopy. A new approach to mapping organic and biological surfaces. Proc. natn. Acad. Sci. U.S.A. 69, 561-565.

Griffith, O.H., Rempfer, G.F. \& Lesch, G.H. (1981) A high vacuum photoelectron microscope for the study of biological specimens. Scanning Electron Microscopy 2, 123-130.

Griffith, O.H., Rempfer, G.F. \& Nadakavukaren, K.K. (1982) Imaging of cell surfaces by photoelectrons. Proc. 10th Int. Congr. Electron Microscopy, Hamburg 1, 59-68.

Griffith, O.H., Nadakavukaren, K.K. \& Jost, P.C. (1984) The potential role of photoelectron microscopy in the analysis of biological surfaces. Scanning Electron Microscopy, 2, 633-644.

Grund, S., Eichberg, J. \& Engel, W. (1979) Photoemission electron microscopy of biological specimens. Beitr. electronenmikroscop. Direktabb. Oberfl. 12, 157-164.

Houle, W.A., Engel, W., Willig, F., Rempfer, G.F. \& Griffith, O.H. (1982) Depth of information in photoelectron microscopy. Ultramicroscopy 7, 371380 .
Hyne, R.V. \& Garbers, D.L. (1982) Inhibition of the guinea-pig sperm acrosome reaction by a low molecular weight factor(s) in epididymal fluid and serum. J. Reprod. Fert. 64, 151-157.

Meizel, S. (1984) The importance of hydrolytic enzymes to an exocytotic event, the mammalian sperm acrosome reaction. Biol. Rev. 59, 125-157.

Mollenstedt, G. \& Lenz, F. (1963) Electron emission microscopy. Adv. Electronics Electron Phys. 18, 251329.

Mrsny, R.J. \& Meizel, S. (1981) Potassium ion influx and $\mathrm{Na}^{+}, \mathrm{K}^{+}-\mathrm{ATPase}$ activity are required for the hamster sperm acrosome reaction. J. Cell Biol. 91, 77-82.

Phillips, D.M. (1977) Mitochondrial disposition in mammalian spermatozoa. J. Ultrastruct. Res. $\mathbf{5 8}$, 144-154.

Rempfer, G.F., Nadakavukaren, K.K. \& Griffith, O.H. (1980) Topographical effects in photoemission microscopy. Ultramicroscopy 5, 437-448.

Schwarzer, R.A. (1981) Emission electron microscopy-A review. Microsc. Acta 84, 5!-86.

Yanagimachi, R. (1969) In vitro acrosome reaction and capacitation of golden hamster spermatozoa by bovine follicular fluid and its fractions. J. exp. Zool. 170, 269-280.

Yanagimachi, R. (1970) The movement of golden hamster spermatozoa before and after capacitation. J. Reprod. Fert. 23, 193-196.

Yanagimachi, R. (1972) Fertilization of guinea pig eggs in vitro. Anat. Rec. 174, 9-20.

Yanagimachi, R. (1981) Mechanisms of fertilization in mammals. In Fertilization and Embryonic Development in vitro, pp. 82-182. Eds L. Mastroianni, Jr \& J. D. Biggers. Plenum Press, New York.

Yanagimachi, R. \& Phillips, D.M. (1984) The status of acrosomal caps of hamster spermatozoa immediately before fertilization in vivo. Gamete Res. 9, 1-19.

Received 6 August 1984 